REGARDS

SUR L'ECONOMIE ALLEMAND

BULLETIN ECONOMIQUE DU CIRAC
Regards sur l'économie allemande

Bulletin économique du CIRAC

$94 \mid 2009$

Varia

\title{
Dorma : une holding de tradition familiale
}

Solène Hazouard

\section{OpenEdition}

\section{Journals}

Édition électronique

URL : http://journals.openedition.org/rea/3994

DOI : 10.4000/rea.3994

ISBN : 978-2-8218-0884-3

ISSN : 1965-0787

Éditeur

CIRAC

Édition imprimée

Date de publication : 1 décembre 2009

Pagination : 33-38

ISSN : 1156-8992

Référence électronique

Solène Hazouard, «Dorma : une holding de tradition familiale », Regards sur l'économie allemande [En ligne], 94 | décembre 2009, mis en ligne le 01 décembre 2011, consulté le 23 avril 2019. URL : http:// journals.openedition.org/rea/3994; DOI : 10.4000/rea.3994 


\section{Dorma : une holding de tradition familiale}

\section{Solène Hazouard}

En octobre dernier, Dorma (Ennepetal) s'est vu décerner un prix récompensant son image de marque lors de la présentation de l'ouvrage Marken des Jahrhunderts qui, d'Adidas à Zeiss, couvre 281 entreprises emblématiques du Made in Germany. Depuis sa création en 1908, l'entreprise familiale, et employeur modèle, est devenue au fil du temps une holding multinationale, élevée au rang de leader mondial sur le marché des techniques de porte, des portes automatiques, des portes vitrées et des murs mobiles. Le groupe est en outre $n^{\circ} 1$ en Allemagne sur son cinquième segment "Sécurité Temps Accès", dédié à la gestion du temps, des issues de secours et au contrôle d'accès.

Malgré une place acquise parmi les grandes entreprises de dimension internationale, la Dorma Holding GmbH \& Co. KGaA demeure fortement tournée vers l'Allemagne. Membre de diverses fédérations professionnelles, ce hidden champion (Simon, 2007) y a établi des réseaux de partenariat avec ses clients B2B, fidélisés à l'aide de mesures spécifiques de formation et de conseil. II entretient par ailleurs un dialogue constant avec des architectes lors de la phase de conception des produits, privilégiant ainsi l'innovation et le design.

Plus spécifiquement, l'entreprise conserve un lien fort avec sa région d'origine, située en Rhénanie du Nord-Westphalie, au cœur de la "Métropole Ruhr ». L'attachement particulier au site historique, au même titre que l'importance accordée au facteur humain et l'enjeu successoral actuel contribuent à inscrire Dorma dans la catégorie des entreprises familiales, typiques du Mittelstand outre-Rhin.

Avec 882,2 millions $€$ de chiffre d'affaires au cours de l'exercice 2008/2009 (clos le 30 juin) et une équipe de plus de 6600 collaborateurs à travers le monde, la holding Dorma est sur le point de quitter le Mittelstand pour rejoindre les grandes entreprises multinationales. Le groupe, qui incluait en 2008 non moins de 87 sociétés, est structuré en cinq grandes divisions établies selon les familles de produits proposées.

Dorma - Évolution du chiffre d'affaires et du nombre de salariés (1999-2009)

\begin{tabular}{|cccc|}
\hline Année & CA (en millions $€$ ) & Évolution $(\%)$ & Salariés (valeur moyenne) \\
$99 / 00$ & 495 & - & 4193 \\
$00 / 01$ & 573 & $+15,8 \%$ & 4767 \\
$01 / 02$ & 613 & $+7 \%$ & 5113 \\
$02 / 03$ & 663 & $+8,2 \%$ & 5590 \\
$03 / 04$ & 649 & $-2,1 \%$ & 5510 \\
$04 / 05$ & 700 & $+7,9 \%$ & 5817 \\
$05 / 06$ & 767 & $+9,6 \%$ & 6149 \\
$06 / 07$ & 838 & $+9,3 \%$ & 6410 \\
$07 / 08$ & 894 & $+6,7 \%$ & 6767 \\
$08 / 09$ & 882 & $-1,3 \%$ & 6780 \\
\hline
\end{tabular}

Source des données : Bilanzpressekonferenz 24.09.09, www.dorma.de

La première, intitulée " techniques de portes », comprend les ferme-portes, poignées et béquillages, serrures, paumelles et charnières commercialisés dans le monde par l'intermédiaire de vendeurs spécialisés. La division « portes automatiques », développée dans les années 1970, représente un moteur important de 
croissance tandis que la division « solutions architecturales du verre » rassemble poignées et plinthes pour portes en verre, ainsi que des installations vitrées complètes. Sur ces trois marchés, qui représentaient respectivement près de $38,5 \%$, $29 \%$ et $16 \%$ du CA du groupe en 2008 , ainsi que sur celui des murs mobiles (12\% du CA global), Dorma occupe le rang de leader mondial, acquis grâce à une stratégie précoce d'internationalisation. Le groupe assure désormais son leadership en misant sur sa supériorité technologique, son offre de solutions complètes et de produits diversifiés ainsi que sur la promotion de son image de marque, à laquelle contribuent conjointement une présence forte à l'international, une gestion de la qualité à l'échelle mondiale et un effectif important dédié au service client, avec plus de 1100 collaborateurs dans le monde, dont 200 sur la zone Allemagne.

Bien que le cinquième segment "Sécurité Temps Accès » (STA) contribue dans une moindre mesure au CA (4,5\%), l'entreprise est tout de même numéro un en Allemagne sur ce marché dédié à la gestion des issues de secours ainsi qu'aux systèmes de contrôle d'accès et de gestion du temps.

\section{Dorma : une tradition familiale centenaire}

En 1908, Rudolf Mankel et son beau-frère Wilhelm Dörken fondent la société en commandite Dörken \& Mankel KG à Ennepetal, dans le Land de Rhénanie du Nord-Westphalie, entre la Ruhr et la Wupper. Dans un premier temps, l'entreprise fabrique des charnières à double action et des vis à têtes fraisées, misant avant tout sur la qualité des produits. Dès 1927, la marque Dorma, issue de la contraction du nom des deux fondateurs, fait l'objet d'un dépôt à l'office des brevets. Comme pour nombre d'entreprises allemandes, l'après 1945 est synonyme d'un nouveau départ : à partir de 1950, Dorma met en production son premier ferme-porte, le TS 50, pour occuper aujourd'hui le rang de leader mondial sur ce marché. C'est également depuis cette époque du « miracle économique " allemand que la société enregistre une croissance annuelle moyenne de $13,5 \%$ de son CA et de $7,2 \%$ de son personnel. A partir de 1962, Dorma élargit son offre aux mécanismes de portes automatiques puis en 1969, KarlRudolf Mankel, le petit fils du fondateur Rudolf Mankel, reprend les rênes de la société Dörken \& Mankel, dont il convertit le nom en Dorma. L'entreprise compte alors près de 500 collaborateurs pour un CA de 20 millions $€$. S'ensuit une phase d'expansion à l'échelle mondiale allant de pair avec une diversification de la gamme de produits. C'est en effet en 1976 que Dorma se lance dans la technique pour porte en verre et fonde en 1977 la filiale Dorma Glas. La même année, l'entreprise choisit la France comme lieu d'implantation de sa première société de distribution à l'étranger.

Malgré son rang d'entreprise multinationale...

..et les questions afférentes d'ordre interculturel,...
Le groupe a fondé sa première filiale étrangère en France en 1977, pour franchir le pas vers l'Asie l'année suivante, avec la création d'une usine de production à Singapour. Bien que nombre de pays asiatiques aient présenté des avantages plus nets en termes de coûts, Dorma a fait le choix d'un site doté d'une main-d'œuvre efficace et parlant couramment anglais, localisé à un endroit stratégique de la route maritime vers l'Asie. Dès lors, la holding n'a cessé de s'étendre à l'échelle mondiale : outre un réseau dense de partenaires commerciaux, œuvrant depuis les quatre coins du globe, la société compte aujourd'hui 71 filiales réparties sur 47 pays, les principaux sites de production étant localisés en Europe, sur le continent américain et dans des pays comme Singapour, la Malaisie ou la Chine.

Bien que le choix de l'Asie puisse s'avérer intéressant en termes de coûts de production, le critère de la demande locale est le plus déterminant : les deux tiers du CA réalisés en Asie sont directement issus de la production des usines asiatiques. De plus, comme le confie le dirigeant des affaires opérationnelles de l'entreprise, Michael Schädlich, "la présence sur ces marchés tire ensuite les produits de fabrication allemande. Cela signifie qu'un client asiatique qui traite avec Dorma pourra acheter la palette complète de nos produits depuis l'Asie. Et une grande partie de cette palette de produits provient d'Europe ou des États-Unis ». A l'échelle mondiale, l'entreprise réalise aujourd'hui $24,3 \%$ de son CA en Allemagne, $38,4 \%$ en Europe et $37,3 \%$ dans le reste du monde.

Son implantation est notable en Inde notamment, où Dorma a développé un système de partenariat de distribution avec des entreprises qui intègrent des produits Dorma aux constructions locales, voire proposent une offre complète de SAV. Cette initiative, qui inclut des mesures de formation, a été récompensée en 2007 lors de la conférence annuelle de la Chambre de commerce germano-indienne à Bonn, en raison de sa contribution au développement des relations économiques entre les deux pays. Car l'enjeu est de taille : à l'horizon 2020, l'Inde devrait se placer au troisième rang des puissances économiques mondiales, derrière les 
États-Unis et la Chine. Par ailleurs, l'Allemagne est aujourd'hui le principal partenaire de l'Inde à l'échelle de l'Union européenne, avec une balance commerciale largement excédentaire $(+2,9$ milliards $€)$, sur un volume d'échanges record de 13,5 milliards $€$ en 2008 (ministère fédéral des Affaires étrangères).

La qualification des entreprises partenaires sous-tend une formation initiale et continue ciblée des collaborateurs de Dorma sur place. A cet égard, Dorma a mis en place une gestion du personnel reposant sur les deux piliers "qualité " et " perspectives » et accorde une attention toute particulière aux différences culturelles subsistant entre la maison-mère et la filiale indienne. Car l'objectif final de Dorma est de pouvoir fidéliser en son sein des collaborateurs motivés et qualifiés.

Bien qu'internationalisé, le groupe Dorma accorde une place prépondérante au site Allemagne, qui concentre les activités intensives en savoirs et orientées vers les services. Comme dans de nombreux groupes allemands internationalisés, les emplois hautement qualifiés et les activités de R\&D demeurent localisés au sein de la maison-mère. C'est en Allemagne que sont notamment accomplis les tests certifiant la qualité de produits fabriqués dans les usines des États-Unis, de Singapour, de Chine, du Brésil ou de France. Car comme l'indique Michael Schädlich : " il s'agit d'une mission typique de la maison-mère, que nous réalisons ici pour le reste du monde, afin de maintenir les standards de qualité de Dorma ". On comprend dès lors que malgré une participation relative au CA du groupe en 2008 (à peine $25 \%$ ), l'Allemagne bénéficiait tout de même de plus de $55 \%$ des investissements et employait près de $40 \%$ du personnel global.

\section{Une culture active de réseau}

Comme toute entreprise allemande, Dorma est affiliée aux fédérations professionnelles comme la Fédération des portes automatiques (Fachverband der Türautomation, FTA), qui compte une douzaine d'entreprises membres. Le groupe appartient également à la Fédération des fabricants et installateurs de systèmes de sécurité (Bundesverband der Hersteller- und Errichterfirmen von Sicherheitssystemen, $B H E$ ), qui rassemble plus de 560 entreprises du secteur. Son codirigeant, Michael Schädlich, représente par ailleurs les intérêts de Dorma au niveau de la branche, au sein de la Fédération des matériaux de construction (Bundesverband Baustoffe - Steine und Erden, BBS), de même qu'au niveau fédéral, en sa qualité de membre du Comité pour le Mittelstand de la Fédération allemande de l'industrie (BDI). Ces multiples affiliations de branche permettent à l'entreprise d'influer sur les conditions cadre qui sont le pivot de la politique des PME outre-Rhin.

Si Dorma mise sur la croissance externe via une politique de fusions-acquisitions efficace, le groupe table également sur son développement en interne, stimulé notamment par l'instauration d'un partenariat stratégique avec ses clients B2B sur la zone allemande, mais aussi en Suisse et en Autriche. Le concept «DORMA System-Partner » créé en 2000, qui rassemble aujourd'hui plus de 700 entreprises artisanales spécialisées dans le verre, le bois, le métal, l'aménagement intérieur et les matériels électriques, a pour visée de développer la coopération entre les différents corps de métiers. Selon une approche gagnant-gagnant, il permet aux partenaires du groupe de s'appuyer sur un soutien professionnel, aussi bien en matière de savoir-faire que dans les domaines de la commercialisation et de la gouvernance d'entreprise. L'aide prodiguée par Dorma peut s'étendre à la communication externe des entreprises, via des moyens de promotion ciblés allant des panneaux de chantiers au développement de sites Internet, mais concerner encore, avec l'aide d'experts, certains aspects juridiques comme le droit de la succession ou la réglementation applicable aux contrats et attributions de marchés dans le secteur de la construction. Les entreprises artisanales s'adaptent en outre aux évolutions du marché à l'aide d'une offre riche de séminaires produits et d'ateliers orientés vers la pratique, complétée par la publication régulière de newsletters.

Au sein de la division "Sécurité Temps Accès ", les formations et conseils dispensés aux partenaires sont accompagnés du service " Dorma Stark », compre-

\section{...le groupe maintient un lien privilégié avec son pays d'origine}

Une société très insérée dans le réseau des fédérations professionnelles

Une stratégie de partenariat pour privilégier à la fois la relation clients sur le long terme... 
...et l'innovation

De nombreuses récompenses

Un attachement fort à la région d'origine du groupe... nant un concept d'exposition PoS (Point of Sale), une boutique Internet B2B de 600 articles, un guide électronique de produits ainsi que le programme de garantie des liquidités PROT€CT. Développée avec le soutien d'experts du crédit, d'assureurs et d'avocats, cette prestation englobe un site d'information, un système de management des créances, l'assurance achats de Dorma, une assurance complète et l'octroi de garanties bancaires.

Dans une perspective de dialogue constant, le groupe travaille conjointement avec des architectes auxquels il propose des formations ciblées (notamment dans les Länder de Rhénanie du Nord-Westphalie, de Saxe et de Hesse, où la formation continue est obligatoire dans ce domaine). Cette politique d'échanges réguliers a permis à l'entreprise de développer une large gamme de produits innovants, qui a valu à Dorma d'obtenir notamment un contrat d'équipement du plus haut gratte-ciel au monde, le Burj Dubai, ou encore le prix international du design « red dot », qui tient non seulement compte du potentiel créatif de l'entreprise, mais aussi de la qualité des produits commercialisés.

En 2006, l'Office fédéral des brevets plaçait Dorma dans le Top 50 des entreprises au regard du nombre de brevets déposés. La même année, le groupe se voyait décerner le label «Top 100 » honorant une cohabitation réussie entre science et marché, particulièrement vectrice d'emploi. Un enjeu de premier ordre au sein d'une économie globalisée, sur lequel a insisté Andreas Pinkwart, ministre en charge de l'Innovation dans le Land de Rhénanie du Nord-Westphalie, à l'occasion de la remise du prix européen "Plus X Award » en 2007, qui récompensait, entre autres, le confort d'utilisation de PORTEO, l'opérateur de porte automatique du groupe Dorma. «Pour les entreprises, l'innovation ne constitue pas la cerise sur le gâteau mais bien la condition sine qua non d'un succès durable. Dans notre société, elle est la source d'emplois hautement qualifiés et pérennes », affirmait-il.

A l'instar de la plupart des entreprises familiales observées par l'institut ZEW (Mannheim) dans une étude de 2008, le groupe demeure attaché à sa région d'origine: les filiales allemandes du holding se concentrent toujours principalement en Rhénanie du Nord-Westphalie. L'activité liée au travail du verre est regroupée au nord-est du Land, avec l'implantation depuis 1987 de la filiale Dorma Glas dans la ville de Bad Salzuflen, et l'acquisition en 2006 à Rietberg, près de Gütersloh, de l'entreprise MAME Türendesign $\mathrm{GmbH}$, leader sur le marché allemand des portes en verre à surface travaillée. Le siège allemand de la division «Sécurité Temps Accès » est situé quant à lui à Bonn, lieu d'implantation de la MBB Gelma $\mathrm{GmbH}$, rachetée par le groupe Dorma en 2000. Car la proximité géographique des différents sites allemands est le résultat d'une stratégie d'acquisitions établie en vue d'exploiter le potentiel industriel du Land, et notamment de la « Métropole Ruhr » : le 1er janvier 1989, le groupe rachète l'entreprise Ogro fondée en 1866 à Velbert. Située à $20 \mathrm{~km}$ à l'ouest d'Ennepetal et à mi-chemin entre Essen et Wuppertal, cette ville constitue depuis la fin du $19^{\mathrm{e}}$ siècle un site de renommée internationale en matière de serrures et ferrures.

\section{Ennepetal : une commune industrielle dans le réseau « Métropole Ruhr »}

Avec ses 33000 habitants et ses $57 \mathrm{~km}^{2}$, qui en font l'une des municipalités les plus étendues géographiquement de Rhénanie du Nord-Westphalie, Ennepetal abrite les entreprises Dorma, Altenloh, Brinck \& CO GmbH \& Co. KG (le fabricant des vis SPAX) et ThyssenKrupp Bilstein Suspension $\mathrm{GmbH}$ (dans le domaine des amortisseurs). La commune s'inscrit dans la « Métropole Ruhr » dont elle est l'une des 53 villes constitutives. Centre industriel du Land de Rhénanie du Nord-Westphalie, la « Métropole Ruhr » se range au troisième rang des agglomérations de l'Union européenne, derrière Londres et Paris.

...et au site d'Ennepetal
Cette politique de rachats vient renforcer l'ancrage local du groupe, dont témoignent les paroles prononcées en 2004 par le propriétaire de l'entreprise KarlRudolf Mankel, lors de l'inauguration sur le site historique d'Ennepetal de nouveaux bâtiments administratifs en présence du chancelier Gerhard Schröder: "Dorma a ses racines ici, à Ennepetal, et restera toujours une entreprise de la région. Avec la construction de ces nouveaux bâtiments, il me tenait à cœur de montrer clairement mon attachement à la région ». 
Dans les années qui ont suivi, plus de 10 millions $€$ ont été investis en vue de moderniser le site de production d'Ennepetal. Un projet défendu ainsi par Michael Schädlich, malgré de plus faibles coûts de production dans l'usine de Singapour, où Dorma avait établi une filiale dès 1978: "nous sommes convaincus que l'équipe, les savoirs, la technologie et les produits nous permettront d'assurer durablement une production de rang mondial sur le site d'Ennepetal ». Pas moins de 3,1 millions $€$ ont été jugés nécessaires pour les travaux de l'atelier de peinture et de finalisation des produits. Conformément au planning initial, ce projet de promotion du site historique de Dorma s'est achevé à la veille de la commémoration du centenaire de l'entreprise. II a permis en outre la restructuration d'usines dans le domaine des techniques de porte et des portes automatiques ainsi que la mise en place d'une nouvelle plate-forme logistique, le « Regional Logistics Center Central Europe » (RLC CE+) sur le site de Wuppertal. Ce choix géographique, motivé par la densité des voies de communication dans cette région du Land, au cœur de la "banane bleue " européenne, et la proximité du site d'Ennepetal, constitue pour Michael Schädlich « un signe clair en faveur du site économique allemand ». Le RLC CE+ dessert les marchés scandinaves, d'Europe centrale et les nouveaux marchés d'Europe de l'Est jusqu'aux portes de l'Asie.

\section{Une culture marquée par la tradition familiale}

Outre-Rhin, Dorma fait partie de ces $93 \%$ d'entreprises familiales qui réalisent à elles seules près de la moitié du CA global et contribuent à hauteur de $57 \%$ à l'emploi des salariés (données ZEW, 2008). Car son propriétaire, Karl-Rudolf Mankel, n'est autre que le petit-fils de l'un des deux fondateurs. Si cette situation est représentative de l'industrie du bâtiment dans son ensemble, constituée à $99 \%$ d'entreprises familiales, elle s'avère en revanche atypique dans les entreprises de plus de 500 collaborateurs toutes branches confondues, où ce taux est de seulement $19 \%$.

Comme le souligne le ZEW, l'engagement social des entreprises familiales, sur lequel celles-ci communiquent d'ailleurs relativement peu, vise avant tout leurs propres collaborateurs. A l'échelle de Dorma, cela se traduit notamment par un programme de formation continue systématique et institutionnalisé, récompensé en 2008 par le prix fédéral « Top Job » dans la catégorie « Développement des Compétences et Perspectives ». Cette distinction, décernée par l'ancien ministre fédéral de l'Économie Wolfgang Clement (il était auparavant ministre de l'Économie, puis Ministre-Président de Rhénanie du Nord-Westphalie) et l'Université St. Gallen, est le fruit d'une politique de recrutement en interne de jeunes managers, formés sur une période de deux ans puis soutenus via un système de supervision de groupe, coachings individuels et apprentissages ciblés, ou encore du programme " Shared Experiences Inside DOrma » (SEIDO), qui offre aux experts de l'entreprise la possibilité de mettre en œuvre les connaissances acquises dans des projets internationaux et de se perfectionner dans leur domaine.

Plus globalement, Dorma a reçu en 2008 pour la quatrième année consécutive le label qualité "Top Job », qui recouvre en outre les catégories "Leadership et Vision », "Culture et Communication », "Axe familial et social », " Management interne " ainsi que « Motivation et Dynamiques ». Outre le versement de primes et bonus accordés en fonction de la performance, Dorma offre des possibilités de travail à temps partiel et des compléments de revenus pour les familles, afin de permettre à ses collaborateurs et collaboratrices de concilier vie familiale et vie professionnelle. Une politique du personnel qui se révèle fructueuse, dans une entreprise qui place la motivation de ses collaborateurs au même rang que la satisfaction clients et dans laquelle, du moins sur les sites allemands, chaque salarié travaille en moyenne 13 ans.

Car le groupe voit son attractivité en tant qu'employeur consacrée par l'obtention en 2009 de deux labels du Handelsblatt Junge Karriere. Le premier, distinguant les meilleurs employeurs à l'échelle fédérale (Top Arbeitgeber), est décerné par la société indépendante Corporate research foundation (CRF). II tient compte des
Une dynamique régionale

Dorma mise sur le capital humain

Un employeur attractif 
Aujourd'hui, l'enjeu de la succession possibilités d'évolution des salariés, de la sécurité de l'emploi, de la culture et de l'image d'entreprise, du type de rémunération et de l'équilibre atteint entre vie professionnelle et familiale. Le second label (Fair Company) souligne la qualité des relations avec étudiants et jeunes diplômés, promouvant un recours équitable aux stages en entreprise. En matière de formation initiale, Dorma propose aux apprentis d'accéder à dix métiers différents, dont six dans les domaines technique et logistique et quatre orientés vers la pratique commerciale et l'informatique.

Dernier phénomène caractéristique des entreprises familiales outre-Rhin : la succession. Afin d'y préparer au mieux son entreprise, Karl-Rudolf Mankel, âgé de 66 ans, a transféré en mars dernier la majeure partie de ses parts sociales à ses deux filles, Christine et Stephanie, alors âgées respectivement de 26 et 23 ans. II est toutefois prévu que la gestion opérationnelle de l'entreprise reste aux mains d'une personne étrangère à la famille Mankel, que celle-ci doit actuellement recruter. Car Michael Schädlich, qui codirige Dorma depuis 1996, quittera l'entreprise au terme de son mandat le 30 juin 2010. Dans le cadre de cette transmission patrimoniale, Karl-Rudolf Mankel et ses filles ont institué l'an passé la fondation Rudolf Mankel Stiftung, dont la mission est de venir en aide aux nécessiteux ainsi qu'aux personnes non endettées se retrouvant dans le besoin. A l'instar de Dorma, plus de 70000 entreprises familiales allemandes se retrouvent chaque année confrontées à l'enjeu de la succession, selon les estimations de l'Institut de recherches sur le Mittelstand IfM (Röhl, 2008). Le ZEW précise par ailleurs qu'en cas d'un départ de l'entrepreneur à 60 ans, près de $21 \%$ de ces entreprises connaîtront un changement de direction dans les 30 ans à venir, dont $90 \%$ ces 15 prochaines années. Si la transmission successorale a lieu au-delà de 65 ans, ce pourcentage se réduit à $12 \%$.

EN CETTE PÉRIODE ÉCONOMIQUE MOUVEMENTÉE, les responsables de la holding familiale Dorma, hissée au rang de hidden champion, demeurent relativement sereins. Car si les conséquences de la crise influeront plus tardivement sur l'industrie du bâtiment, qui réagit habituellement aux variations conjoncturelles avec un décalage temporel de six mois à un an, et malgré le fait que les aides des programmes conjoncturels fédéraux, portant notamment sur la rénovation des bâtiments, soient accordées à ce jour avec une grande parcimonie, la PME multinationale considère la crise comme une chance. Outre les possibilités de rachats, que permet un ratio de fonds propres particulièrement élevé $(62,5 \%)$, et une réduction substantielle des coûts (-22,2 millions $€$ au sein des filiales en 2008/09), la direction de Dorma a réitéré lors d'un entretien l'importance capitale des activités de réseau : " en temps de crise, le partenariat gagnant-gagnant établi avec nos clients et fournisseurs soutient la confiance à long terme et renforce notre collaboration ». Une stratégie parachevée par la recherche constante de nouveaux débouchés : afin de se positionner sur le marché résidentiel et semi-professionnel, le groupe mise désormais sur l'aménagement intérieur.

\section{Indications bibliographiques}

- Bourgeols I., «Entreprises familiales : un rôle clef outre-Rhin », Regards sur l'Economie Allemande, $\mathrm{n}^{\circ} 82 / 2007$

- Bourgeois I., «Rhénanie du Nord-Westphalie: reconversion du charbon au high-tech », in BouRgeoIs I. (dir.), Allemagne : compétitivité et dynamiques territoriales, CIRAC, Cergy-Pontoise, juillet 2007

- Broer E., huber S., Kirchdörfer R., Peters B., Rotfuß W., Schmidt S, Schröder M., WESTERHEIDE P., Wirtschaftliche und gesellschaftliche Bedeutung von Familienunternehmen, Abschlussbericht an das Bundesministerium für Wirtschaft und Technologie, ZEW, Mannheim, octobre 2008

- RöHL K.-R., « Die Zukunft der Familienunternehmen in Deutschland - Potenziale und Risiken in der globalen Wirtschaft », IW Analysen, Deutscher Instituts-Verlag, Cologne, 2008

- Simon H., Hidden Champions des 21. Jahrhunderts-Die Erfolgsstrategien unbekannter Weltmarktführer, Campus Verlag, Francfort, 2007

- www.auswaertiges-amt.de ; www.baustoffindustrie.de ; www.bdi.eu ; www.bhe.de ; http://business. metropoleruhr.de ; www.dorma.de ; www.ebundesanzeiger.de ; http://www.fta-online.de ; www.crf.com/ germany ; www.karriere.de ; www.nrwinvest.com. 